\title{
Eficácia de placas educativas no descarte de resíduos sólidos urbanos e à não alimentação do sagui-de-trufo-preto (Callithrix penicillata)
}

\author{
Effectiveness of educational signs in the disposal of urban solid waste and the non-feeding of black \\ truffle marmosets (Callithrix penicillata) \\ Efectividad de la cartelería educativa en la disposición de residuos sólidos urbanos y la no \\ alimentación de titíes de trufa negra (Callithrix penicillata)
}

\author{
Isa Lucia de Morais \\ ORCID: https://orcid.org/0000-0001-8748-9723 \\ Universidade Estadual de Goiás, Brasil \\ E-mail: isamorais1@gmail.com \\ Cristiane Daia Rizzo \\ ORCID: https://orcid.org/0000-0002-0802-7369 \\ Faculdade de Quirinópolis, Brasil \\ E-mail: cristiane.rizzo@hotmail.com \\ Suzi Mari Brandelero \\ ORCID: https://orcid.org/0000-0002-9551-6995 \\ Universidade Estadual de Goiás, Brasil \\ E-mail: suzibrandelero@yahoo.com.br \\ Wellington Hannibal \\ ORCID: https://orcid.org/0000-0001-7141-1243 \\ Universidade Estadual de Goiás, Brasil \\ E-mail: wellingtonhannibal@gmail.com
}

\begin{abstract}
Resumo
Neste estudo, investigamos a eficácia de placas educativas na conscientização da população de Quirinópolis com relação ao descarte de resíduos sólidos e à não alimentação de Callithrix penicillata no Jardim Botânico da Universidade Estadual de Goiás. Avaliamos as diferenças no peso total dos resíduos antes e após a implantação das placas educativas, e as interações entre os fatores: uso da placa [antes, após] com o tipo de resíduo, e testamos a associação entre o peso dos resíduos com a escala temporal para cada tipo de resíduo. Houve uma diminuição no peso de papel, plástico e vidro no interior; e papel, plástico e metal no entorno do Jardim Botânico, e não foi observada a presença de alimentos para os indivíduos de saguis após o uso das placas educativas. Esses resultados corroboram a eficácia das placas educativas. Entretanto, mais pesquisas são necessárias para averiguar se esse padrão se mantém em outros cenários de Educação Ambiental.
\end{abstract}

Palavras-chave: Comunicação visual; Educação ambiental; Jardim Botânico.

\begin{abstract}
In this study, we investigated the effectiveness of educational boards in raising awareness among the population of Quirinópolis regarding the disposal of solid waste and non-feeding of Callithrix penicillata in the Botanical Garden of the State University of Goiás. We evaluated the differences in the total weight of waste before and after implementation of the educational signs, and the interactions between the factors: use of the sign [before, after] with the type of residue, and we tested the association between the weight of the residues with the time scale for each type of residue. There was a decrease in the weight of paper, plastic and glass inside; and paper, plastic and metal in the surroundings of the Botanical Garden, and the presence of food for the individuals of marmosets was not observed after the use of the educational plates. These results support the effectiveness of educational boards. However, more research is needed to find out if this pattern is maintained in other Environmental Education scenarios.
\end{abstract}

Keywords: Visual communication; Environmental education; Botanical Garden.

\section{Resumen}

En este estudio se investigó la efectividad de las juntas educativas en la sensibilización de la población de Quirinópolis sobre la disposición de residuos sólidos y no alimentación de Callithrix penicillata en el Jardín Botánico de la Universidad Estadual de Goiás. Evaluamos las diferencias en el total peso de los residuos antes y después de la implementación de los letreros educativos, y las interacciones entre los factores: uso del letrero [antes, después] con el tipo de residuo, y probamos la asociación entre el peso de los residuos con la escala de tiempo para cada tipo de 
residuo. Hubo una disminución en el peso del papel, plástico y vidrio en el interior; y papel, plástico y metal en los alrededores del Jardín Botánico, y no se observó presencia de alimento para los individuos de titíes luego del uso de los platos educativos. Estos resultados apoyan la efectividad de los consejos educativos. Sin embargo, se necesita más investigación para averiguar si este patrón se mantiene en otros escenarios de Educación Ambiental.

Palabras clave: La comunicación visual; Educación ambiental; Jardín Botánico.

\section{Introdução}

A geração desenfreada de Resíduos Sólidos Urbanos (RSU), a falta de conscientização por parte da maioria das pessoas e a disposição de forma incorreta desses materiais geram impactos ambientais, os quais, consequentemente, afetam a saúde da população (Gouveia, 2012; Fontes et al. 2021; Freeman, 2021; Leite et al., 2021). Nas últimas décadas, além do acréscimo na quantidade, os RSU passaram a abrigar em sua composição elementos sintéticos e perigosos aos ecossistemas e à saúde humana, em virtude das novas tecnologias incorporadas ao cotidiano. Os RSU são materiais ou substâncias, sólidas ou semissólidas, gerados por atividades industriais, domésticas, hospitalares e de serviços de varrição. Podem ser definidos e segregados de acordo com: a matéria que os constitui, a sua formação, as atividades que lhes deram origem e de acordo com os impactos que podem causar à saúde e ao meio ambiente (ABNT NBR 10.004, 2004; Brasil, 2010).

No Brasil, entre 2010 e 2019, a quantidade de RSU coletados cresceu 24\% e atingiu 72,7 milhões de toneladas, dos quais aproximadamente $60 \%$ seguiram para disposição final adequada em aterros sanitários. No entanto, a realidade da disposição inadequada ainda está presente em todas as regiões do país, tendo aumentado $16 \%$ em relação a 2010 . Essa situação traz consequências danosas para o meio ambiente e para a saúde das pessoas, que tem sofrido a cada ano com esse incremento de volume de resíduos depositados em locais inadequados, contaminando o solo, o ar e os recursos hídricos (Abrelpe, 2020; Santos et al., 2020; Gadelha et al., 2021; Leite et al., 2021).

Neste viés, foi aprovada a Lei $n^{\circ} 14.026$, de 15 de julho de 2020, em sincronia com o ideal de que urge a implementação de ações práticas para realizar uma boa gestão do saneamento como plataforma para o desenvolvimento dos municípios e, principalmente, visando assegurar a sustentabilidade da presença de todos nós no planeta. A referida lei, chamada de novo marco regulatório do saneamento básico, estimula a concorrência, as concessões do setor e a privatização de empresas públicas estatais de saneamento, entre outras inovações importantes no intuito de solucionar os graves problemas ambientais e de saúde pública causados pela insuficiência de saneamento no Brasil (Abrelpe, 2020; Gadelha et al., 2021).

Entretanto, no âmbito das inúmeras políticas públicas e ações institucionais em prol de reverter esse quadro quanto aos graves problemas ambientais no tocante ao saneamento básico (Gadelha et al., 2021) é imprescindível a realização de uma proposta concreta que conduza à sensibilização da população sobre a problemática em questão (Garcia et al., 2019). Se não houver sensibilização, não haverá novas formas de pensar e tampouco de agir. Logo, uma possibilidade de conciliação entre a sensibilização ambiental e a gestão dos resíduos sólidos é a Educação Ambiental (EA), a qual fornece a cada pessoa ou coletivo um melhor entendimento quanto ao seu papel enquanto sujeito crítico e atuante em relação ao meio ambiente. A EA auxilia a população a tornar-se mais responsável, engajando-a a atuar de forma coletiva com inovações no universo das questões ambientais para que conheça seus direitos e deveres, se torne crítica e criativa e, também, promova, por meio da sustentabilidade, uma melhor qualidade de vida local e globalmente (Friede et al., 2019; Silva \& Oliveira, 2020; Santos et al., 2021).

Entre as estratégias de ações no âmbito da EA está, em diferentes níveis de contexto e atuação, a inserção de placas educativas no sentido de desenvolver uma capacidade de efetiva comunicação socioambiental e assegurar a manutenção do fluxo dessa comunicação com seus públicos, com a intencionalidade de educar ambientalmente pela defesa do meio ambiente e qualidade de vida. Assim, as placas educativas integram a comunicação visual para orientação no espaço urbano e público (na maioria dos casos) e são direcionadas para influir no comportamento dos cidadãos quanto ao cuidado com a coisa pública e 
com o direito do próximo (Costa, 1987; Silva \& Saraiva, 2020; Fontes et al., 2021). Neste viés, é possível incluir as placas educativas como uma das ferramentas de educomunicação porque trata-se de uma comunicação popular, a qual é considerada bem público e que se manifesta desde a criação do material até a sua veiculação, com a premissa central o diálogo, a capacidade de comunicar algo entre todos os envolvidos em uma determinada especificidade em que se encontram, de forma a emergir não só em espaços formais, mas sobretudo fora deles (Freitas \& Ferreira, 2020).

Entretanto, embora seja usual a inserção de placas educativas em programas de EA, essa abordagem raramente tem sido avaliada quanto à sua eficácia e ou aplicada na conscientização sobre a geração de RSU (veja Almeida; Bueno, 2017) e à não alimentação de animais silvestres. Portanto, neste estudo, investigamos a eficácia de placas educativas na conscientização da população de Quirinópolis com relação ao descarte de resíduos sólidos e à não alimentação do sagui-de-tufo-preto (Callithrix penicillata) no Jardim Botânico da Universidade Estadual de Goiás (JBUEG), Câmpus Sudoeste, Sede Quirinópolis. Especificamente, avaliamos as diferenças no peso total de RSU antes e após a implantação das placas educativas, e as interações entre os fatores: uso da placa [antes, após] com o tipo de resíduo [papel, plástico, vidro e metal]. Nós também testamos a associação entre o peso dos RSU com a escala temporal para cada tipo de resíduo.

\section{Material e Métodos}

A presente pesquisa tem abordagem descritiva e quantitativa e suporte metodológico de acordo com o proposto por Pereira et al. (2018). Segundo esses autores, nessa metodologia faz-se a coleta de dados quantitativos por meio do uso de medições de grandezas e obtém-se por meio da metrologia, números com suas respectivas unidades, resultando em um conjunto de dados, os quais podem ser analisados pelo pesquisador por meio de técnicas matemáticas como é o caso das porcentagens, estatísticas e probabilidades, métodos numéricos, métodos analíticos e geração de equações e/ou fórmulas matemáticas aplicáveis a algum processo.

\section{Jardim Botânico da Universidade Estadual de Goiás - Quirinópolis}

O estudo foi realizado no JBUEG, Câmpus Sudoeste - Sede: Quirinópolis (Figura 1), localizado na Avenida Brasil, no Conjunto Hélio Leão, no município de Quirinópolis, GO. Esse município está inserido na microrregião 18 de mesmo nome, ao sul do estado de Goiás e possui uma área de $3.792 \mathrm{~km}^{2}$ e população de 50.065 habitantes (IBGE, 2019).

O JBUEG tem $17.112 \mathrm{~m}^{2}$ e o plantio das espécies arbóreas no local iniciou-se em 1995. Desde que foi criado, os objetivos do JBUEG foram o de ser um banco de espécies vegetais, resguardando exemplares típicos do Cerrado em área urbana, e o de subsidiar estudos práticos de botânica e ser fonte de pesquisa pela comunidade acadêmica do curso de Ciências Biológicas, Geografia e áreas afins, da UEG.

No JBUEG existem 97 espécies, das quais 83\% são nativas, distribuídas em 26 famílias e 66 gêneros, com 1.803 indivíduos jovens e 487 adultos (Cruz, 2019). Esse espaço é importante para área urbana de Quirinópolis, pois é o maior agrupamento vegetacional da cidade. Assim, essa vegetação serve de local de pouso, nidificação e forrageamento por várias espécies de aves, além da importância para a manutenção de vários outros táxons da fauna local. Como exemplo, vale ressaltar a presença de um grupo de saguis (Callithrix penicillata) que encantam os moradores no entorno do JBUEG e visitantes, principalmente as crianças (Resende, 2012). 
Figura 1. Fotografia aérea do Jardim Botânico da Universidade Estadual de Goiás, Câmpus Sudoeste, Sede Quirinópolis (espaço entre a linha amarela).

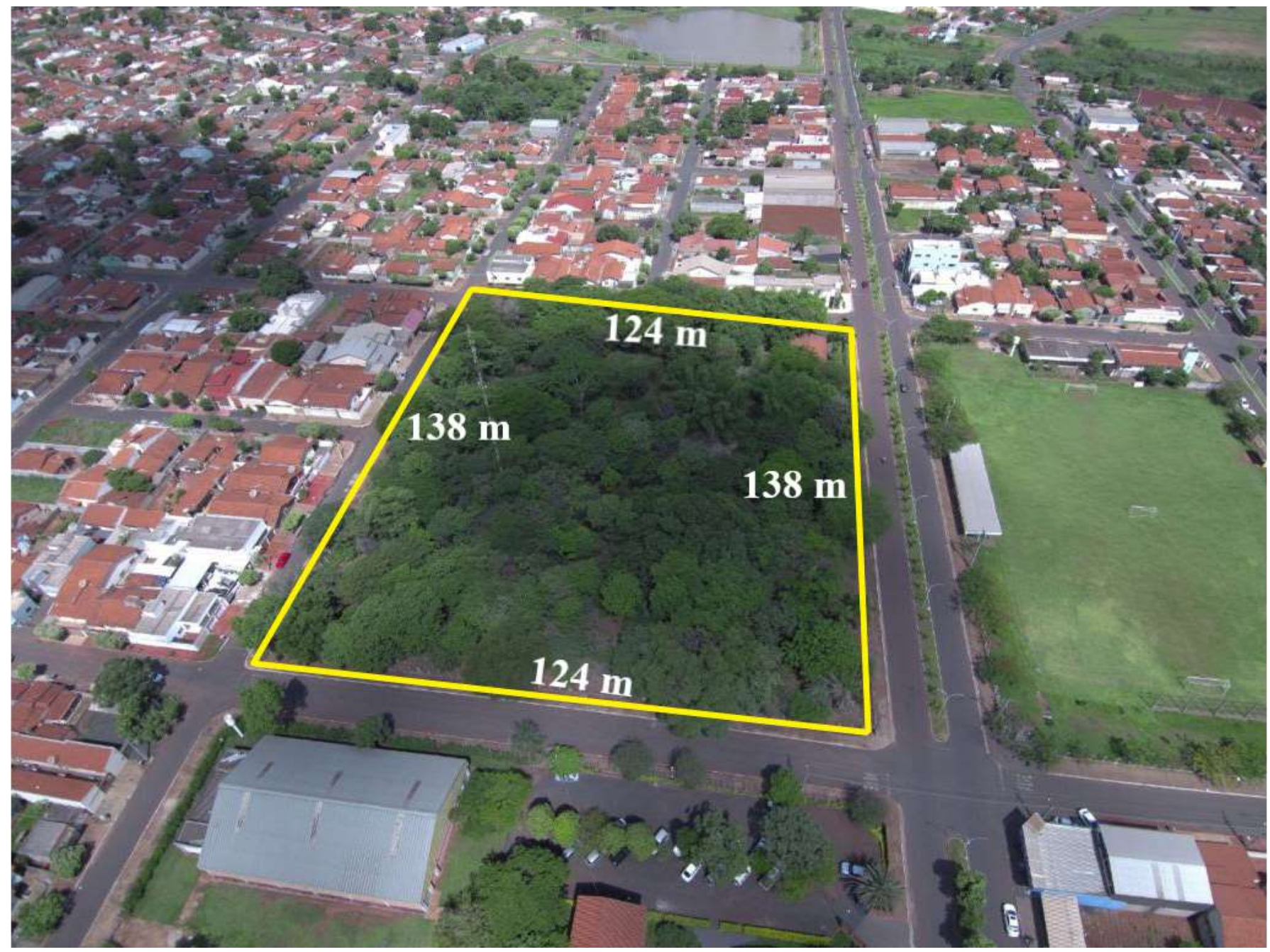

Fotografia: Lucas Leão.

Enquanto área verde, o JBUEG desempenha inúmeras funções que beneficiam os munícipes no seu entorno, direta ou indiretamente. Entre estas funções está a ecológica resultante da manutenção da biodiversidade no espaço urbano e dos serviços ambientais prestados pela vegetação e o solo não impermeabilizado. Logo, esse ambiente promove melhorias na qualidade do ar, água e solo e no clima dos bairros nas suas proximidades (Resende, 2012).

Inerente à educação ambiental existe uma Trilha Ecológica no JBUEG cujo percurso abrange o conhecimento sobre espécies do Cerrado como nomes vulgares e científicos, uso econômico, medicinal e alimentício, entre outras curiosidades. A criação da trilha fez parte de um trabalho de conclusão de curso lato sensu de Oliveira (2016). Ela é continuamente visitada por inúmeros estudantes do ensino básico, técnico e superior de Quirinópolis. Isso contribui para ampliar o conhecimento das espécies do Cerrado e a valoração do JBUEG. Neste viés, o espaço do JBUEG permite uma gama de possibilidades de atividades de extensão, ensino e pesquisa (Resende, 2012). Na última década tem crescido o número de pesquisas e utilização da área pelos acadêmicos dos Cursos de Ciências Biológicas (Vidigal \& Resende, 2011; Maia, 2012; Silva, 2012; Silva, 2014; Costa et al., 2016) e lato sensu (Oliveira, 2016; Cruz, 2019) da UEG.

Entretanto, embora o JBUEG seja uma área verde e de relevante importância ecológica, educativa e para qualidade de vida dos quirinopolinos (Vidigal \& Resende, 2011) esse espaço tem sido local de descarte de RSU por moradores no entorno e transeuntes. O JBUEG não dispõe de nenhum funcionário da universidade, específico para a remoção dos RSU tanto no 
interior quanto no calçamento. A retirada dos RSU é feita ocasionalmente durante as visitas de alunos das instituições de ensino na Trilha Ecológica, sendo uma das atividades do percurso a coleta dos RSU; e quando os agentes de saúde (servidores municipais) fazem mutirão no JBUEG para eliminar criadouros propícios à reprodução do mosquito da dengue (Aedes aegypti). Outro aspecto negativo observado é a alimentação fornecida aos indivíduos de saguis pelos moradores.

\section{Placas educativas}

Confeccionamos 20 placas ( $60 \mathrm{~cm}$ de comprimento por $40 \mathrm{~cm}$ de largura cada), com cinco frases educativas, sendo uma frase em cada placa. Assim, foram produzidas quatro repetições de placas (Figura 2). Cada frase teve o intuito de despertar no cidadão a percepção de que o JBUEG é uma área verde importante para a manutenção da biodiversidade e a saúde dos munícipes. A arte que integrou cada uma das placas também foi planejada para ter uma estética em harmonia com a vegetação. Fixamos cinco placas com as diferentes informações em cada lado da quadra, distante $20 \mathrm{~m}$ uma da outra, e a 1,6 m do solo.

Figura 2: Esquema das cinco placas educativas (a-e) fixadas no alambrado do Jardim Botânico da Universidade Estadual de Goiás, Câmpus Sudoeste, Sede Quirinópolis.
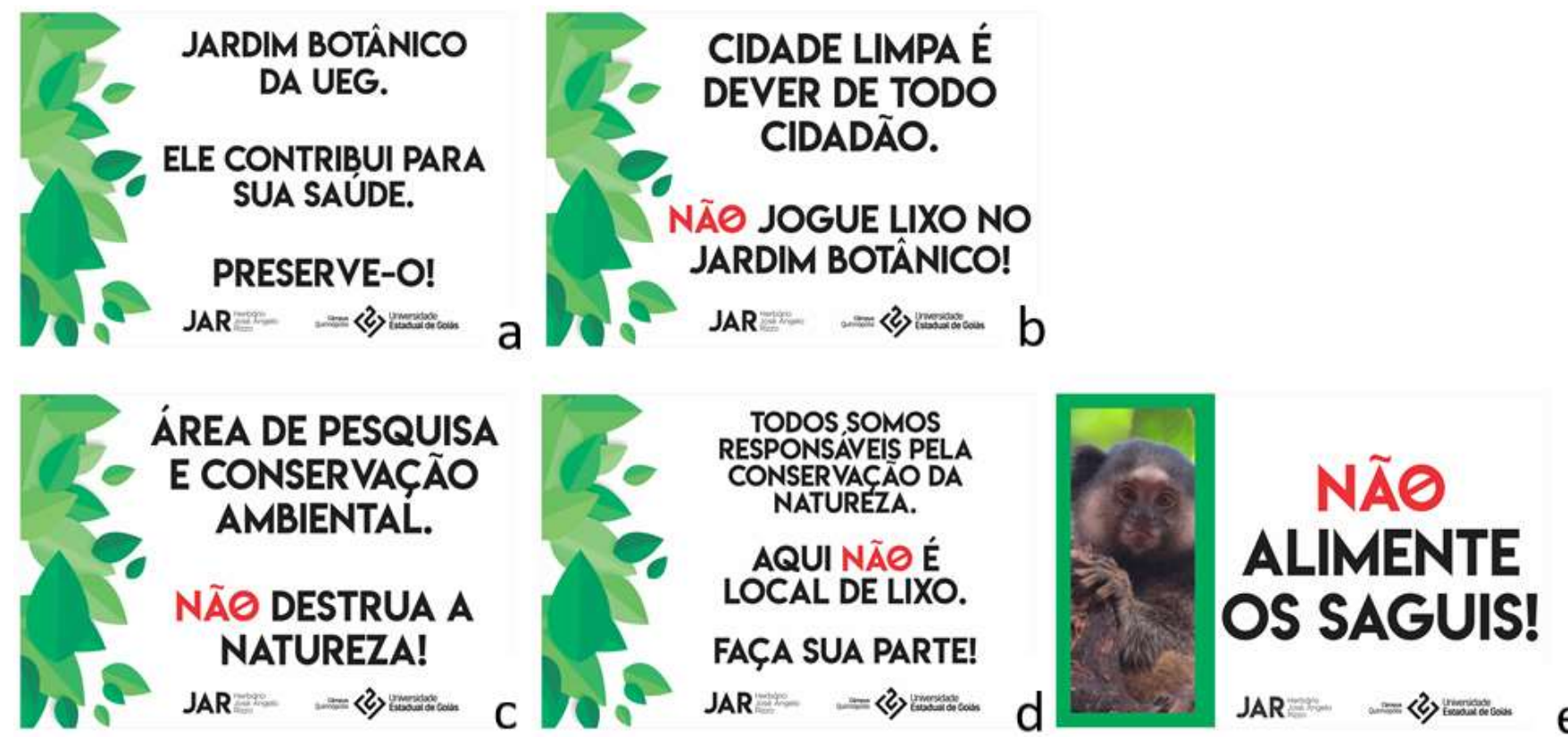

Fonte: Autores.

\section{Coleta de dados}

Para investigarmos a importância de atividades de educação ambiental na conscientização da população sobre o descarte de resíduos sólidos, realizamos a coleta e pesagem dos RSU dentro e no entorno do JBUEG, mensalmente em: janeiro, fevereiro e março (antes da instalação das placas), e em abril, maio e junho (após a instalação das placas).

Coletamos os resíduos no último dia de cada mês, por meio de caminhadas não sistematizadas dentro e fora do JBUEG, de forma que o intervalo entre as coletas fosse de aproximadamente 30 dias. Ao longo dessas caminhadas todos os resíduos encontrados eram colocados em sacos plásticos e levados para o laboratório para posterior triagem e pesagem. $\mathrm{Na}$ triagem separamos os RSU de acordo com o tipo de resíduo (Quadro 1) e, em seguida, pesamos usando uma balança semianalítica calibrada e nivelada. 
A avaliação quanto à eficácia da placa educativa específica para a não alimentação dos indivíduos de saguis (Figura 2e) foi restrita à observação cotidiana quanto ao comportamento das pessoas quanto a este aspecto. Como a entrada no Jardim Botânico é restrita e necessita de agendamento, as pessoas só conseguem alimentar esses animais nas proximidades do alambrado, sendo o usual elas deixarem frutas, principalmente bananas, no alambrado.

Quadro 1: Tipos de resíduo sólidos urbanos e sua caracterização.

\begin{tabular}{|ll|}
\hline $\begin{array}{l}\text { Tipo de } \\
\text { resíduo }\end{array}$ & \multicolumn{1}{c|}{ Caracterização } \\
\hline Papel & Jornais, revistas, caixas e embalagens. \\
Plástico & Embalagens de garrafas, garrafões, frascos, baldes, sacolas, canos, entre outros objetos de plástico. \\
Vidro & Garrafas, copos, cacos, potes, entre outros objetos de vidro. \\
Metal & $\begin{array}{l}\text { Objetos diversos feitos de metais podendo ser de alumínio, ferro ou aço, entre outros, como latas } \\
\text { de alimentos e bebidas, panelas, copos, por exemplo. }\end{array}$ \\
Outros & $\begin{array}{l}\text { Roupas, isopor, resíduos de eletrodomésticos, fibras sintéticas, borracha, entre outros que não se } \\
\text { enquadram nos outros quatro tipos. }\end{array}$ \\
\hline
\end{tabular}

Fonte: adaptado da Lei 12.305 de 2010.

\section{Análise dos dados}

Para investigarmos a eficácia das placas educativas, juntamos os dados dos pesos (em gramas) do interior e entorno do JBUEG, então, comparamos esses valores dentro de cada tipo de resíduo [papel, plástico, vidro e metal] antes e após a implantação das placas. A interação entre o uso da placa [antes, após] com o tipo de resíduo [papel, plástico, vidro e metal] no interior e entorno do JBUEG foi avaliada pelo teste de Qui-quadrado, função chisq.test. A associação entre o peso dos RSU com a escala temporal foi avaliada pela correlação de Pearson, função cor.test. Todas as análises foram executadas pelo programa R (R Core Team, 2019).

\section{Resultados e Discussão}

Um total de $59.385 \mathrm{~g}$ de resíduos (37.120 g [antes] e $22.265 \mathrm{~g}$ [após] o uso das placas) foram coletados durante toda a fase de monitoramento no interior e entorno do JBUEG. Os RSU no entorno e interior do JBUEG, quanto aos riscos potenciais ao meio ambiente, pertencem à classe II, sendo, portanto, não perigosos (ABNT, 2004). Embora a área interna do JBUEG seja maior que o seu entorno (fora do alambrado) a quantidade de RS coletada no entorno foi maior (Figura 3). Os RSU coletados no interior do JBUEG se localizavam mais próximos das laterais, aproximadamente cinco metros adentro, a partir do alambrado. Já a maioria dos RSU coletada no entorno do JBUEG se localizava próxima do alambrado, em uma faixa não pavimentada com cerca de um metro de largura, contígua ao alambrado em todo o entorno do JBUEG, entre o alambrado e o calçamento. A maior concentração dos RSU próxima ao alambrado, principalmente no exterior do JBUEG, se justifica pelo fato deste ser o local de maior facilidade de acesso para os moradores e ou transeuntes descartarem esse material.

Após a fixação das placas educativas houve uma redução na maioria dos tipos de RSU coletados no entorno e interior do JBUEG. Entre os tipos de RSU que aumentou a quantidade após a instalação das placas está o metal e o isopor (incluído em outros) no interior do JBUEG e o vidro no entorno do JBUEG (Figura 3). Apesar disso, não encontramos uma interação entre o uso da placa com o tipo de resíduo do interior $\left(\chi^{2}=0,94 ; \mathrm{gl}=4 ; \mathrm{p}=0.92\right)$ e entorno $\left(\chi^{2}=2,21 ; \mathrm{gl}=4 ; \mathrm{p}=0.69\right)$ do JBUEG. Mas, houve uma diminuição no peso de papel, plástico e vidro no interior; e papel, plástico e metal no entorno, após o uso das placas educativas (Figura 3), o que corrobora a eficácia dessa ferramenta de EA. Considerando que o tipo de RSU que 
aumentou é oriundo de latinhas de alumínio e garrafas de vidro, a maioria destas constituída por embalagens de bebidas alcóolicas, estas podem ter sido descartadas por transeuntes e não por moradores nas proximidades do JBUEG.

Figura 3. Peso em gramas de cada tipo de resíduo sólido urbano coletado no interior e entorno do Jardim Botânico da Universidade Estadual de Goiás, antes e depois da implantação das placas educativas.

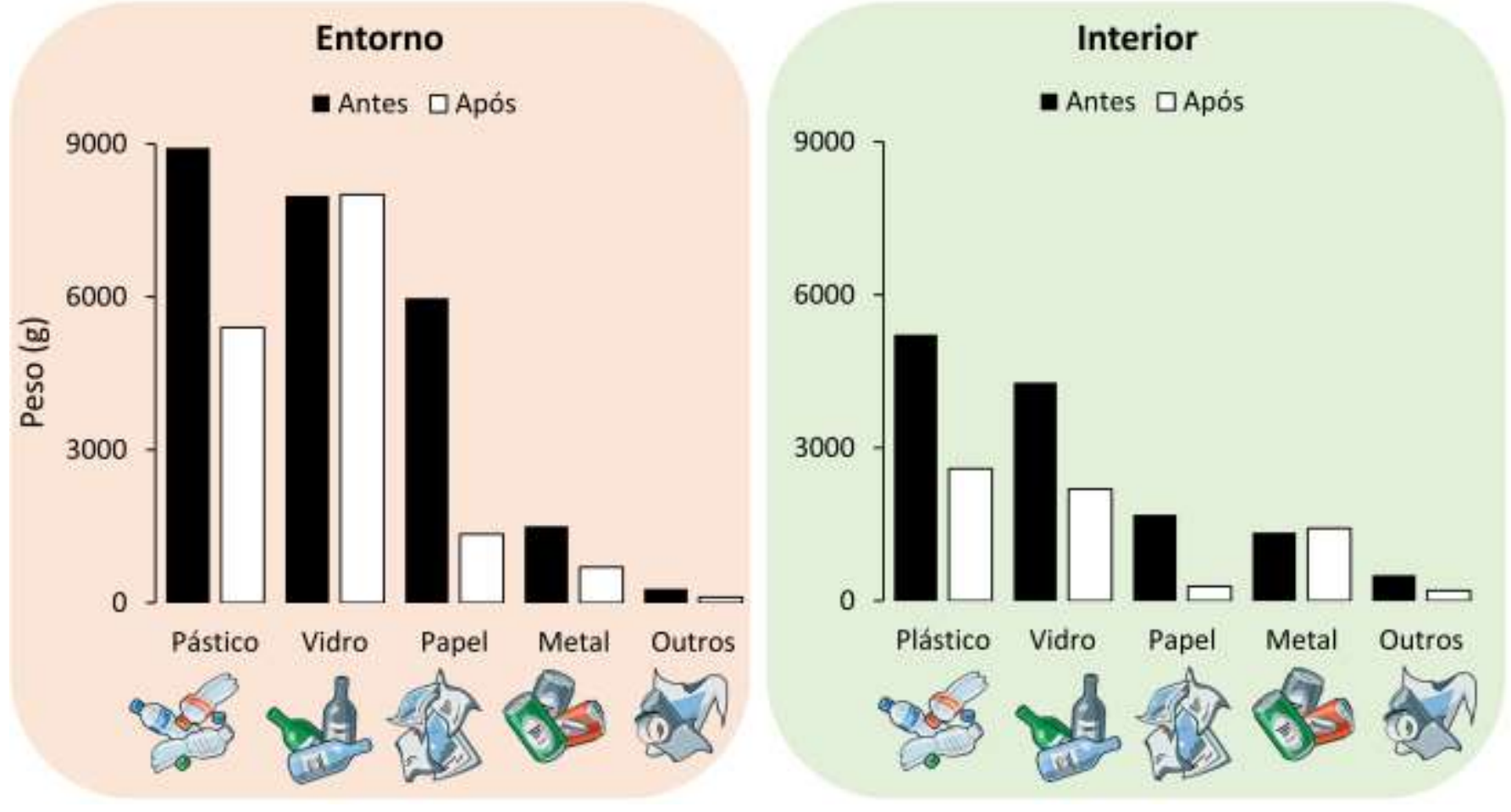

Fonte: Autores.

A maior quantidade de RSU encontrada compreendeu os tipos plástico e vidro. O plástico é material inerte e sua decomposição na natureza pode se estender a 100 anos ou mais. Esse resíduo corresponde a 16,8\% dos RSU do Brasil (Abrelpe, 2020). Ele consegue poluir em todos os estágios de seu ciclo de vida, desde quando o petróleo e o gás são extraídos para produzi-lo até quando é descartado indevidamente, depositado em aterros, reciclado de maneira equívoca ou queimado (Montenegro; Vianna \& Teles, 2020).

A participação do plástico entre os materiais que compõem os RSU no Brasil ainda é pequena quando comparada à dos países desenvolvidos, mas vem aumentando, principalmente com a pandemia de Covid-19. O Brasil é o $4^{\circ}$ maior gerador de resíduos plásticos do mundo (Kaza et al., 2018). Em 2020 foram descartados 13,35 milhões de toneladas de plásticos no país (Abrelpe, 2020). Segundo dados do Atlas do Plástico, na vida moderna tocamos mais em plástico do que em nossos entes queridos. Em cada pedido de delivery, compra em supermercado ou até em máscaras descartáveis e computadores, o plástico está cada vez mais presente em nossas vidas. São diferentes tipos (da garrafa PET ao PVC, de vestidos a embalagens) e muitos são descartáveis, de uso único, que em menos de um mês se tornam resíduos. Esses resíduos contaminam nossas vidas e o meio ambiente (Montenegro; Vianna \& Teles, 2020).

Já o vidro coletado no JB consistiu, principalmente, de garrafas de bebidas alcoólicas. O vidro corresponde a 2,7\% dos RSU no Brasil (Abrelpe, 2020) e apenas 45\% do vidro é reciclado no país. Apesar dos resíduos vítreos possuírem alto índice de reciclabilidade, a maioria é descartada em locais impróprios, e, ao considerarmos a demora para esse resíduo se decompor, ele pode servir de nichos para diversos vetores de doenças ao longo de todo esse tempo. As embalagens de vidro 
podem ser totalmente reaproveitadas no ciclo produtivo, sem nenhuma perda de material e sem poluição para o meio ambiente (Abividro, 2019).

Acompanhando a tendência de que o peso de cada tipo de RSU no interior e entorno do JB tem diminuído após o uso das placas, nossa associação entre o peso dos resíduos com a escala temporal mostrou uma relação negativa superior a $70 \%$ para papel, plástico e vidro do interior do JBUEG, e de 90\% para papel e 60\% para plástico no entorno do JBUEG (Figura 4).

Figura 4. Relação entre o peso dos tipos de resíduos sólidos com a escala temporal, coletados no interior e entorno do Jardim Botânico da Universidade Estadual de Goiás.
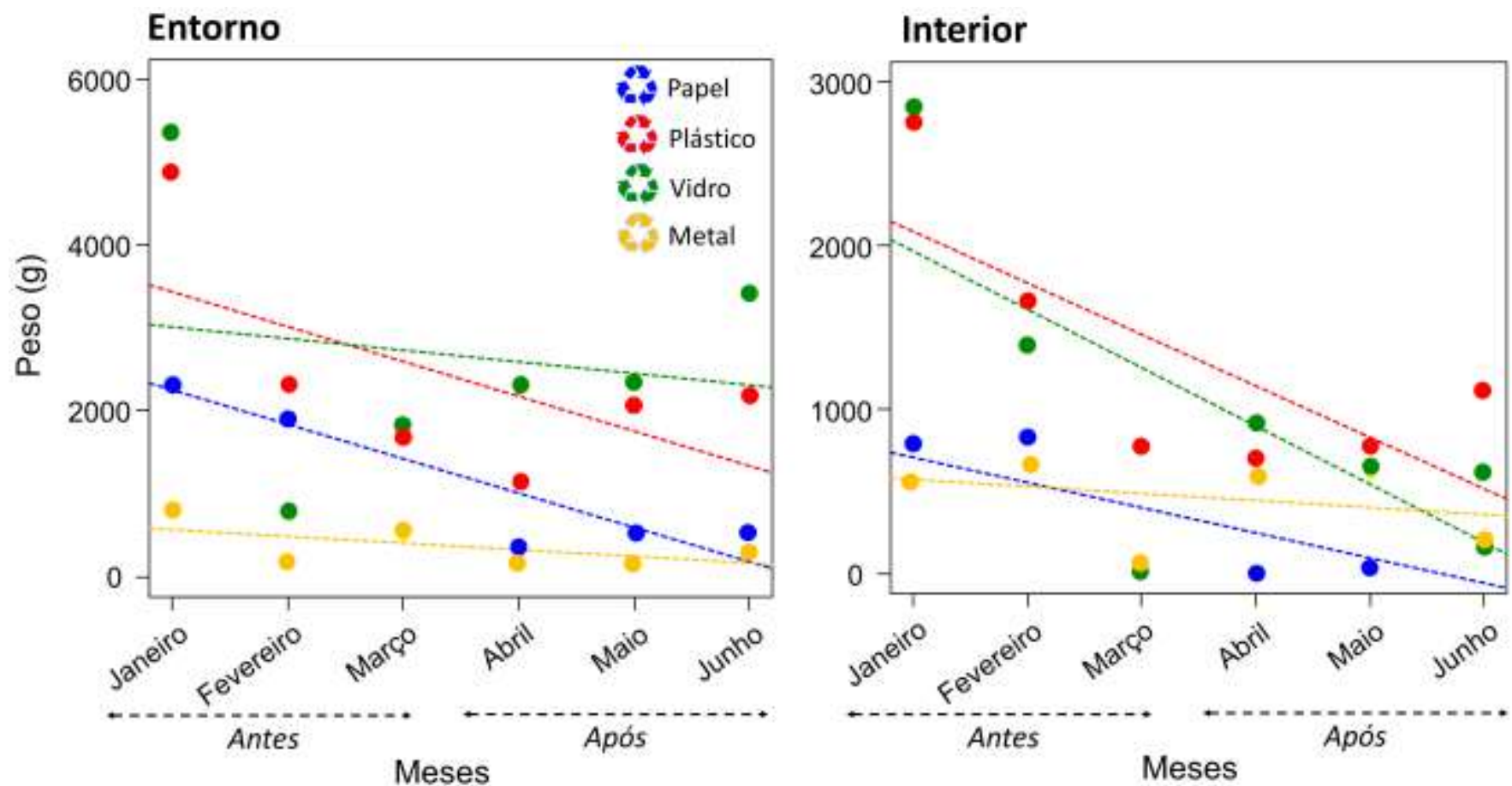

Fonte: Autores.

Na pesquisa de Almeida e Bueno (2017), a qual objetivou identificar as ações de educação ambiental desenvolvidas no Parque Estadual da Serra do Mar - Núcleo de São Sebastião, de modo a buscar minimizar os impactos gerados pela visitação na Praia Brava de Boiçucanga, especificamente aqueles vinculados à geração de resíduos, as pesquisadoras constataram que a maioria dos visitantes ignora as placas informativas e interpretativas e descarta seu lixo na trilha ou deixa na praia. Silva e Liporone (2011) afirmam que embora existam falhas no sistema de gerenciamento de coleta de resíduos, nota-se também que a população tem contribuído para seu descarte inadequado. Mesmo em casos nos quais existem áreas apropriadas para deposição dos RSU, eles apontam que a mudança do comportamento popular é, portanto, um passo fundamental para solução deste problema.

Corroborando com eles Araújo e Pimentel (2016) afirmam que mesmo ciente das consequências do descarte inadequado dos RSU, alguns moradores insistem em descartar seus resíduos em locais inadequados, como praças e canteiros, "a fim de se livrar" de um problema que é de sua responsabilidade. As mesmas autoras ainda colocam que a solução para tal questão não depende apenas de atitudes governamentais ou decisões de empresas, mas que deve partir do empenho de cada cidadão, uma vez que esse tem o poder de recusar produtos potencialmente impactantes, participar de organizações não governamentais ou simplesmente segregar resíduos dentro de casa, facilitando assim os processos de reciclagem.

Os consumidores são atores importantes da cadeia de resíduos, pois são os detentores dos produtos finais e primeiros responsáveis pela correta destinação do resíduo. O artigo $35^{\circ}$ da PNRS determina que é obrigação dos consumidores 
acondicionar adequadamente e de forma diferenciada os resíduos sólidos gerados e disponibilizar adequadamente os resíduos sólidos recicláveis e reutilizáveis (BrasiL, 2010). A partir do descarte adequado pelo consumidor outros atores podem atuar e dar a correta destinação para cada tipo de resíduo.

Quanto à eficácia da placa específica para a não alimentação dos indivíduos de saguis-de-tufo-preto, observamos que após a inserção das placas os moradores no entorno do JBUEG não colocaram mais comida no alambrado para esses animais. Esse resultado foi um aspecto ambiental positivo porque não se deve interferir no ciclo de vida de animais silvestres e, principalmente, na sua dieta alimentar. Para algumas espécies o período de maior índice de natalidade ocorre em épocas de maior abundância de alguns alimentos que serão importantes para o crescimento e perpetuação da espécie (Faria, 2011). Então, a suplementação alimentar (aquela fornecida pela população) pode alterar a dinâmica populacional desses animais, levando a um crescimento exagerado da população e a um desequilíbrio na ecologia urbana local, forçando medidas mitigatórias indesejáveis, como o controle populacional. Apesar das pessoas considerarem que esses animais pareçam estar com fome, e por este motivo se sentirem incumbidos de alimentá-los. Na natureza os saguis dispõem de uma grande variedade de itens alimentares (e.g., artrópodes, frutos e exsudatos [resina das árvores]) e passam a maior parte do tempo em locomoção, descanso e forrageando (Lacher et al., 1984; Vilela \& Faria, 2004; Vilela \& Del-Claro, 2011).

\section{Considerações Finais}

Houve uma diminuição no peso de RSU dos tipos papel, plástico e vidro no interior; e papel, plástico e metal no entorno do JBUEG, e não foi observada a presença de alimentos para os indivíduos de saguis após o uso das placas educativas. Esses resultados corroboram a eficácia dessa ferramenta de EA. Entretanto, mais pesquisas sobre a eficácia das placas educativas são necessárias para averiguar se esse padrão se mantém em outros cenários de EA.

Para futuros estudo recomendamos a análise da percepção da comunidade sobre o descarte inadequado de RSU no JBUEG e a importância desse espaço enquanto área verde urbana. Sugerimos, ainda, o monitoramento do local quanto ao descarte inadequado de RSU e à não alimentação dos saguis, e a ampliação da utilização da Trilha Ecológica presente no local visando aprimorar os conhecimentos dos alunos sobre o meio ambiente e desenvolver a sensibilização ambiental, especialmente em relação à gestão de resíduos sólidos e à valoração das áreas verdes urbanas.

\section{Agradecimentos}

Agradecemos à Universidade Estadual de Goiás pela manutenção do Jardim Botânico da UEG, Câmpus Sudoeste, Sede Quirinópolis e ao Divino Ferreira Marques e Egan Bishop pela doação do valor gasto com a confecção das placas educativas.

\section{Referências}

ABNT (Associação Brasileira de Normas Técnicas). NBR 10004: 2004. 71p.

ABRELPE (Associação Brasileira de Empresas de Limpeza Pública e Resíduos Especiais). Panorama dos Resíduos Sólidos no Brasil 2020. (2020). <https://abrelpe.org.br/panorama-2020>.

ABVIDRO (Associação Técnica Brasileira das Indústrias Automáticas de Vidro). (2019). <https://abividro.org.br/wp-content/uploads/2019/01/Abividro-GuiaReciclagem-do-Vidro.pdf>.

Almeida, L. G. de \& Bueno, F. P. (2017). Educação Ambiental e Geração de Resíduos: a Visitação na Praia Brava de Boiçucanga - Parque Estadual da Serra do Mar, Núcleo de São Sebastião/SP. Revista Eletrônica Uso Público em Unidades de Conservação, 5 (9), 36-51.

Araújo, K. K. \& Pimentel, A. K. (2016). A problemática do descarte irregular dos resíduos sólidos urbanos nos bairros Vergel do Lago e Jatiúca em Maceió, Alagoas. Revista Gestão \& Sustentabilidade Ambiental, 4 (2), 626-668.

Brasil, Lei n. 12.305 de 2010. (2010). Política Nacional dos Resíduos Sólidos. <http://www.planalto.gov.br/ccivil_03/_ato2007-2010/2010/lei/112305.htm>. Costa, J. (1987). Señalética. De la señalización al diseño de programas. Barcelona, Ediciones CEAC. 
Costa, G. F. da, Barros, S. T., Rocha, A. F. S. \& Costa, R. R. G. F. (2016). Comparação do desempenho no viveiro e no campo de progênies de Eugenia calycina Cambess. Revista Mirante, 9 (1), 169-188.

Cruz, N. V. da. Inventário da flora lenhosa e de palmeiras do Jardim Botânico da UEG, Câmpus Quirinópolis, Goiás. (2019). Trabalho de Conclusão (Especialização em Cultura, Diversidade e Meio Ambiente) - Universidade Estadual de Goiás, Câmpus Quirinópolis, Quirinópolis, 2019.

Faria, A. R. G. (2011). Manejo Alimentar e Nutricional de Animais Selvagens para Centros de Triagem.

Fontes, K. D. S. A., Castro, A. C. L. de, Ferreira, T. E. D., Panarelli, E. A. (2021). A compostagem como instrumento de educação ambiental em escolas do Município de João Monlevade - MG. Research, Society and Development, 10 (10), e410101018863.

Freeman, H. R. (2021). O princípio da equidade intergeracional na logística reversa dos resíduos sólidos. In Direito Ambiental e Resíduos Sólidos: perspectiva (inter)nacional transdisciplinar dos 17 ODS da ONU (53-64). Navida Editora.

Freitas, J. V. de \& Ferreira, F. N. (2020). Educomunicação Socioambiental como estratégia pedagógica no ensino infantil. Educação \& Formação, 5 (14), $54-$ 72 .

Friede, R., Reis, D. S., Avelar, K. E. S. \& Miranda, M. G. de. (2019). Coleta seletiva e Educação Ambiental: reciclar valores e reduzir o lixo. Educação \& Formação, 4 (11), 117-141.

Gadelha, H. S., Marques, T., Bezerra Neto, F. C., Lima, M. F. A., Almeida, R. S. de, Marcolino Neto, J., Soares, J. F., Nóbrega, J. C. S., Oliveira, J. D. S. \& Maracajá, P. B. (2021). O novo marco regulatório do saneamento básico e o direito ao acesso à água. Research, Society and Development, 10 (11), e569101118843.

Garcia, E. V., Viesba, L. M. \& Rosalen, M. S. (2019). Educação Ambiental para a sustentabilidade: formação continuada em foco. Humanidades e tecnologia (FINOM), 1 (16), 10-24.

Gouveia, N. (2012). Resíduos sólidos urbanos: impactos socioambientais e perspectiva de manejo sustentável com inclusão social. Ciência \& Saúde Coletiva, 17 (6), 1503-1510.

Instituto Brasileiro De Geografia E Estatística - IBGE. (2019). IBGE Cidades: Quirinópolis, GO. <https://cidades.ibge.gov.br/brasil/go/quirinopolis>.

Kaza, S., Yao, L. C., Bhada-Tata, P. \& Van Woerden, F. (2018). What a Waste 2.0: A Global Snapshot of Solid Waste Management to 2050. The World Bank, 295 p.

Lacher, T. E., Fonseca, G. A. B., Alves-Jr, C. \& Magalhães-Castro, B. (1984). Parasitism of trees by marmosets in a central Brazilian gallery forest. Biotropica, 6 (3), 202-209.

Leite, V. D., Lopes, W. S., Sousa, J. T. de, Albuquerque, M. V. C., Paula e Silva, M. C. C. de, Cartaxo, A. S. B. \& Dantas, G. D. (2021). Resíduos sólidos urbanos no Estado da Paraíba e o contexto da sustentabilidade Ambiental. Research, Society and Development, 10 (1), e33110111781.

Maia, E. G. (2012). Ocorrência de galhas em Quirinópolis, GO e um estudo de caso em Acacia polyphylla DC (Fabaceae). Trabalho de Conclusão (Graduação) - Curso de Ciências Biológicas, Universidade Estadual de Goiás, Câmpus Quirinópolis, Quirinópolis, 2012.

Montenegro, M., Vianna, M. \& Teles, D. B. (2020). Atlas do plástico - fatos e números sobre o mundo dos polímeros sintéticos 2020. Fundação Heinrich Böll e Break Free From Plastic. Edição brasileira.

Oliveira, D. F. de. (2016). Educação Ambiental no Jardim Botânico da UEG, Câmpus Quirinópolis. Monografia (Especialização) - Curso de Educação Para Ciências e Humanidades, Universidade Estadual de Goiás, Câmpus Quirinópolis, Quirinópolis, 2016.

Pereira, A. S., Shitsuka, D. M., Parreira, F. J. \& Shitsuka, R. (2018). Metodologia da pesquisa científica. UFSM. URL <https://repositorio.ufsm.br/bitstream/handle/1/15824/Lic_Computacao_Metodologia-Pesquisa-Cientifica.pdf?sequence=1>.

R Core Team. (2019). R: A language and environment for statistical computing. R Foundation for Statistical Computing, Vienna, Austria. URL $<$ https://www.R-project.org/.2019>.

Resende, I. L. M. (2012). Flora da área urbana de Quirinópolis, Goiás. In: Urzedo, M. F. A. (Org.). Quirinópolis: Mão e olhares diferentes II. 1. ed. Goiânia: Kelps, 349-163.

Santos, P. C., Rocha, A. C. M. A. da, Lima, E. D. S., Santos, J. P. O., Gonzaga, K. S., Cartaxo, P. H. A., Santos, A. S., Bulhões, L. E. L. \& Morais, W. R. S. (2020). Geração de resíduos sólidos urbanos: aplicação de um indicador de sustentabilidade em um município do semiárido alagoano. Research, Society and Development, 9 (8), e244985661.

Santos, G. L. dos, Stuepp, C. A., Campos, S. X. de \& Fragoso, R. O. (2021) Arborização urbana e Educação Ambiental: estudo de caso com o evento "Arboriza Ponta Grossa". Research, Society and Development, 10 (10), e79101018332.

Silva, C. B. da \& Liporone, F. (2011). Deposição Irregular de Resíduos Sólidos Domésticos em Uberlândia: algumas considerações. Revista Eletrônica de Geografia, 2 (6), 22-35.

Silva, M. N. da. (2012). Avifauna do Jardim Botânico da UEG Quirinópolis. TCC (Graduação) - Curso de Ciências Biológicas, Universidade Estadual de Goiás, Câmpus Quirinópolis, Quirinópolis, 2012.

Silva, Q. P. da. (2014). Diversidade de artrópodes de solo em uma área verde urbana de Quirinópolis, GO. Trabalho de Conclusão (Graduação) - Curso de Ciências Biológicas, Universidade Estadual de Goiás, Câmpus Quirinópolis, Quirinópolis, 2014. 
Research, Society and Development, v. 10, n.13, e300101321463, 2021

(CC BY 4.0) | ISSN 2525-3409 | DOI: http://dx.doi.org/10.33448/rsd-v10i13.21463

Silva, J.O.R. \& Oliveira, M.S. (2020). Arborização urbana e a educação ambiental como fator conscientizador. ScientiaGeneralis, 1(2), 1-10. URL $<$ http://scientiageneralis.com.br/index.php/SG/article/view/24>.

Silva, J. S. da \& Saraiva, E. de S. (2020). A importância da Sequência Didática (SD) na Educação Ambiental: um relato de experiência no Centro de Educação em Tempo Integral Monsenhor Boson. Research, Society and Development, 9 (6), e156963505.

Vidigal, M. C. \& Resende, I. L. M. (2011). Florística e fenologia do componente arbóreo do Jardim Botânico da UEG, UnU-Quirinópolis. In: XII SIMPÓSIO DE INICIAÇÃO CIENTÍFICA, 2011, Quirinópolis. Anais... Quirinópolis: UEG.

Vilela, A. A. \& Del-Claro, K. (2011). Feeding behaviour of the black-tufted-ear marmoset (Callithrix penicillata) (Primata, Callithrichidae) in a tropical Cerrado savanna. Sociobiology, 58 (2), 1-6.

Vilela, S. L. \& Faria, D. S. de. (2004). Seasonality of the activity pattern of Callithrix penicillata (Primates, Callithrichidae) in the Cerrado (scrub savanna vegetation). Brazilian Journal Biology, 64 (2), 363-370. 